\title{
Lecciones aprendidas en el control del tabaquismo en España
}

\author{
Esteve Fernández PhD,(1) Joan R. Villalbí PhD, (2) Rodrigo Córdoba PhD. ${ }^{(3)}$
}

\section{Fernández E,Villalbí JR, Córdoba R. Lecciones aprendidas en el control del tabaquismo en España. Salud Publica Mex 2006;48 supl I:SI48-SI54.}

\section{Resumen}

En España ha sido visible la creciente implicación de la sociedad civil en la demanda de políticas de control del tabaquismo. Las bases para la creación del Comité Nacional para la Prevención del Tabaquismo (CNPT) fueron asentadas en 2004.A finales de ese año la intensa actuación para concretar las acciones reguladoras del incipiente Plan Nacional para la Prevención del Tabaquismo culminó en una ley que permitió un salto cualitativo, aprovechando la trasposición legal de la directiva europea de publicidad: la Ley 28/2005, que, en un clima de amplio consenso político y mediático, dispone medidas sanitarias frente al tabaquismo y regula la venta, el suministro, el consumo y la publicidad de los productos del tabaco. El objetivo declarado por esta ley es evitar el inicio del consumo de tabaco particularmente entre los jóvenes, garantizar el derecho de los no fumadores a respirar aire sin humo del tabaco y hacer más fácil el abandono de este hábito a las personas que lo deseen. Sus temas principales son la prohibición de la publicidad del tabaco y la limitación de su consumo en centros de trabajo y espacios públicos cerrados. La nueva ley ha substituido a la normativa previa en España, que era una de las más permisivas de la Unión Europea en temas como la venta de tabaco, limitación de la publicidad y restricciones de lugares de consumo. No obstante, está claro que aún queda mucho por hacer. En este momento hace falta generar apoyo social a la nueva regulación y hacer un esfuerzo importante de sensibilización y educación del público.

Palabras clave: control del tabaquismo; regulación; España
Fernández E,Villalbí JR, Córdoba R.

Lessons learned from tobacco control in Spain. Salud Publica Mex 2006;48 suppl I:SI 48-SI 54.

\begin{abstract}
The growing involvement in Spain by civil society in the demand for tobacco control policies has been notable. The basis for the creation of the National Committee for Tobacco Prevention was established in 2004. At the end of that year, an intensive intervention was aimed at specifying, in law, the regulartory actions in the National Plan for Tobacco Prevention. This would facilitate a qualitative leap, taking advantage of the legal transposition of the European directive on advertising. With broad political consensus, the Law 28/2005 was established regarding sanitary measures for tobacco and the regulation of the sale, supply and consumption of tobacco products. The objective stated in this law is to prevent the initiation of tobacco consumption, especially among youth, guarantee the right of non-smokers to breathe air free from tobacco smoke and make quitting this habit easier for people who wish to do so. The main issues included are the prohibition of tobacco advertisiing and the limitation of tobacco consumption in common work areas and enclosed public spaces. The new law has replaced the previous rules in Spain, which were some of the most permissive in the European Union in terms of tobacco sales, advertising limitations and restrictions on smoking locations. It is clear that there is still much to be done.At this time, more social support needs to be generated in favor of the new regulations, and an important effort needs to be made to educate the public.
\end{abstract}

Keywords: tobacco control; regulation; Spain

(I) Servicio de Prevención y Control del Cáncer, Institut Català d'Oncologia, L'Hospitalet del Llobregat, Cataluña, España. Departamento de Ciencias Experimentales y de la Salud, Universitat Pompeu Fabra, Barcelona, Cataluña, España

(2) Agència de Salut Pública de Barcelona, Cataluña, España, Bloomberg School of Public Health, Johns Hopkins University, Baltimore, USA

(3) Centro de Salud Universitario Delicias Sur, Servicio Aragonés de Salud, Zaragoza, España, Departamento de Medicina, Universidad de Zaragoza, España

Fecha de aceptado: 24 de abril de 2006

Solicitud de sobretiros: Dr. Esteve Fernández, Institut Català d'Oncologia, Servicio de Prevención y Control del Cáncer. Av. Gran Vía s/n Km 2,7,08907 L'Hospitalet, Cataluña, España.

Correo electrónico: efernandez@ico.scs.es 
$\mathbf{E}$ n España, las tendencias de mortalidad por cáncer de pulmón y otras enfermedades relacionadas con el tabaco crecieron hasta fechas recientes, en las que hubo oportunidad de apreciar un primer declive. ${ }^{1-3}$ En la actualidad, se atribuyen unas 54000 muertes anuales al tabaquismo ${ }^{4}$ y cerca de 3000 al tabaquismo pasivo. ${ }^{5}$ Aunque se dispone sólo de encuestas nacionales con métodos estandarizados para toda España desde 1987, en fecha reciente se reconstruyeron los datos hasta la primera mitad del siglo XX (figura 1). ${ }^{6}$ En los hombres la prevalencia en 1945 fue de 42.4\% y aumentó hasta 59.1\% en 1975; el consumo se estabilizó alrededor de 59\% entre 1975 y 1985, momento a partir del cual empezó una disminución progresiva, hasta 48.9\% en 1995. Los datos de la última Encuesta Nacional de Salud del año 2003 establecen la prevalencia de consumo diario en hombres en $37.6 \%$. En las mujeres fue inferior a 5\% hasta inicios de los años setenta, década en que comenzó a aumentar para llegar a 10.1\% en 1975. La prevalencia de consumo diario de cigarrillos en mujeres aumentó de manera sostenida hasta $22.5 \%$ en 1995, si bien parece estabilizarse en los últimos años. Los datos de la última Encuesta Nacional de Salud del año 2003 establecen la prevalencia de consumo diario en mujeres en $24.7 \%$.

En España, la incorporación masiva de las mujeres al consumo de tabaco empezó a finales de la déca- da de los sesenta e inicio de los setenta, ${ }^{7,8}$ en particular a expensas de mujeres con estudios universitarios o medios; no obstante, hoy en día no existen diferencias en el patrón de inicio por razones de género, aunque es más alto en los grupos socioeconómicos desfavorecidos. ${ }^{9}$ Están presentes, además, diferencias sociales apreciables en el patrón de abandono. Aunque la proporción de abandono aumentó en los hombres, la tendencia a hacerlo es más marcada en aquéllos de mayor grado educativo, mientras que en las mujeres la proporción de abandono sólo aumentó en las universitarias de mediana edad..$^{10}$ Por todo ello, se considera el tabaquismo como un problema de salud emergente en mujeres. ${ }^{11}$ Datos recientes en España indican, asimismo, diferencias según el grado educativo o la clase social en el éxito de los tratamientos especializados para abandonar el consumo de tabaco. ${ }^{12}$

En España, el estado actual de difusión del tabaquismo se corresponde con el final de la fase III del modelo descriptivo de la epidemia de tabaquismo. ${ }^{13}$ En dicho modelo se aprecia la clasificación teórica de las poblaciones en diferentes fases o etapas y se puede interpretar, además, en relación con la "teoría de difusión de las innovaciones" de Rogers. ${ }^{14} \mathrm{Al}$ inicio de la fase I, el tabaquismo es excepcional y aumenta, en primer lugar, en hombres, en particular en los de clases aventajadas. En la siguiente etapa (fase II) el consumo

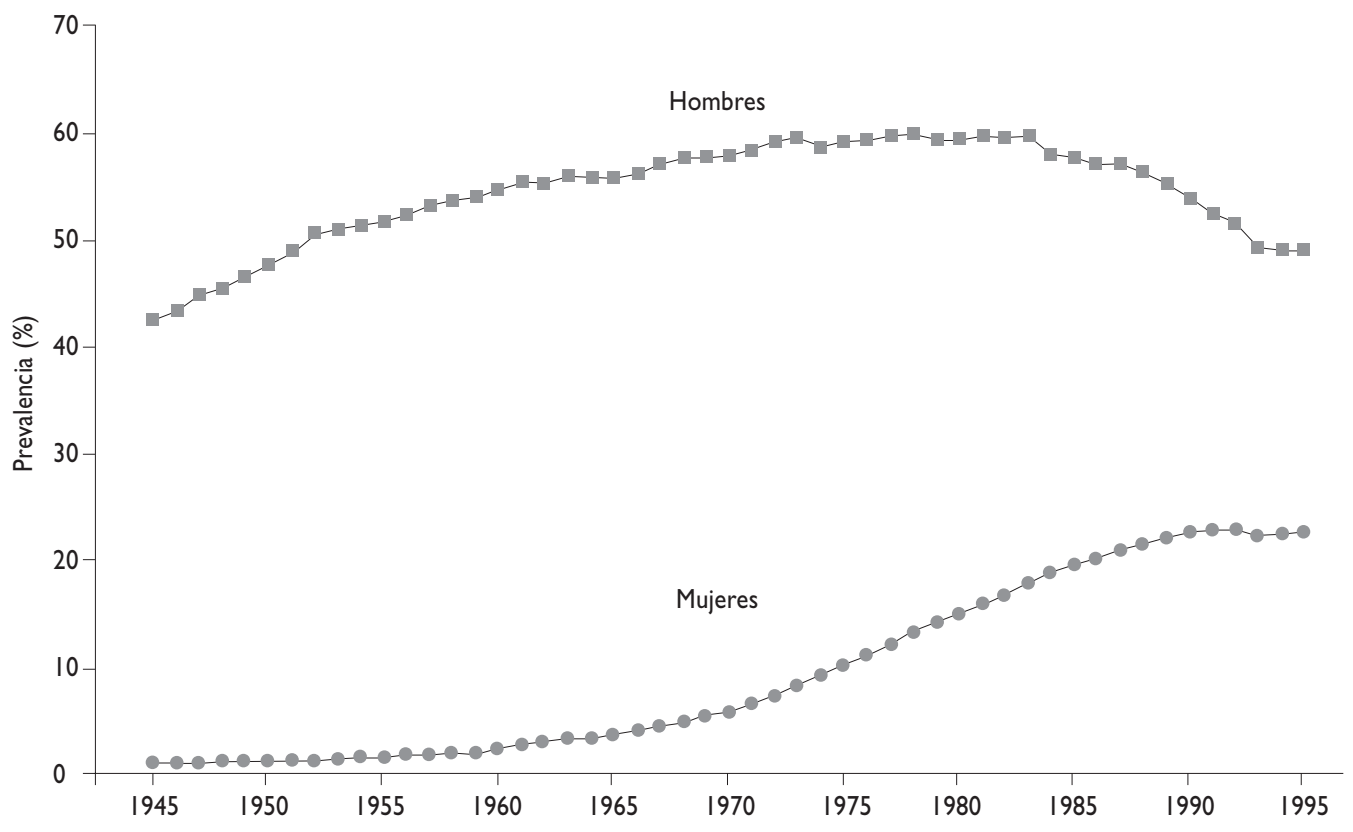

Fuente: Referencia 6

Figura I. Reconstrucción de la prevalencia (\%) de tabaquismo en la población de $\geq$ I6 años en España (1945-1995) 
de tabaco se hace más prevalente, sobre todo en hombres y con aquéllos de mayor estrato social y empieza el aumento de la prevalencia en mujeres. Durante la fase III la prevalencia en hombres comienza a reducirse, sobre todo en aquéllos de mayor posición socioeconómica, mientras que las mujeres, que llevan un retraso de entre 20 y 30 años respecto a los hombres, alcanzan la máxima prevalencia. En la fase IV la prevalencia del consumo se reduce en hombres y mujeres, y en ambos sexos es más elevado en las clases sociales desfavorecidas. Así, en términos generales, el tabaquismo empieza a extenderse en los grupos más favorecidos y después prosigue en los menos aventajados económicamente, sucede en primera instancia en los hombres con una posterior incorporación de las mujeres, como ocurre también con otros comportamientos de acuerdo con la "teoría de difusión de las innovaciones". ${ }^{14}$

\section{El movimiento de prevención del tabaquismo en España}

Hace ya algunos lustros que la ciencia demostró de forma incontrovertible el daño que el tabaco produce en la salud. En tiempos más recientes se demostró también el daño que produce el humo ambiental de tabaco. Todo este conocimiento se trasladó a la sociedad, de modo que en los países desarrollados se redujo mucho el consumo de tabaco y se adoptaron regulaciones que favorecen esta reducción mediante políticas como las fiscales o el control de la publicidad. ${ }^{15} \mathrm{La}$ industria tabaquera se ha resistido a este proceso, $\mathrm{y}$ están bien documentados sus esfuerzos por influir en los políticos y a veces en los científicos, y sobre todo por manipular a los medios de comunicación de modo que no se vieran afectados sus intereses -también en España. ${ }^{16}$ Mientras que algunos países consiguieron incorporar políticas preventivas a la acción del gobierno, acelerando de ese modo el proceso de cambio de la epidemia tabáquica, otros tuvieron menos éxito en este campo y progresan más lentamente. Hasta hace poco, ésta era la situación española. La emergencia de un movimiento organizado contribuyó a cambiar esta situación.

La sociedad civil juega un papel fundamental en el proceso de avance en el control del tabaquismo. ${ }^{17}$ El esfuerzo por traducir los conocimientos sobre el tabaco y el daño que causa en políticas de prevención debe enfrentarse, sin embargo, a obstáculos importantes, por lo que se precisa una presión constante para vencer estas resistencias, imposible sin cierto grado de organización. ${ }^{18}$ En una sociedad democrática el apoyo social permite superar resistencias a la implantación de políticas públicas. Sin este apoyo las presiones de los grupos de interés, como los de la industria tabaquera, sobre los gobiernos frenan el progreso; son presiones que se ejercen de modo directo o a través de los medios de comunicación -presentan el control del tabaquismo como algo controvertido tanto desde la perspectiva social como de la política- y difunden la idea de que tales medidas tendrían impactos negativos sobre el empleo o la recaudación fiscal.

En efecto, en 1989, Phillip Morris (PM) encargó una encuesta que dio como resultado que $73 \%$ de la población española apoyaría una regulación del consumo de tabaco en lugares públicos. Esta demanda social se contuvo de todas las formas posibles durante años a través de un plan estratégico que quedó al descubierto en un documento de PM que se desclasificó por orden judicial en los juicios de Minnessota de 1998. El documento era el "Plan de Aceptabilidad Social" y la línea estratégica del informe representaba, en el fondo, un formidable chantaje sociológico que tuvo profunda penetración en la sociedad. Ese chantaje se resumía en que había que "proteger la armonía social", dado que las evidencias sobre el riesgo del tabaquismo pasivo eran discutibles y los riesgos mínimos comparados con los inconvenientes de regular el consumo en lugares públicos. El objetivo era crear un cuerpo de opinión para que las autoridades se condujeran de manera muy cautelosa y temerosa respecto de las restricciones del tabaco y promover un clima en el cual el fumar representara un acto establecido con firmeza en la sociedad a pesar de sus efectos en la salud. ${ }^{19,20}$ No hubo un movimiento de prevención, todavía muy débil, que contrarrestara este plan y ello retrasó la entrada del control del tabaquismo en la agenda política durante una década.

En España ha sido ostensible la creciente implicación de la sociedad civil en la demanda de políticas de control del tabaquismo. Su principal componente son diversas organizaciones relacionadas con profesionales de la sanidad, pero también participan otros agentes. ${ }^{21}$ Entre éstos se encuentran las organizaciones de víctimas del tabaco o afectados por su uso (como los laringectomizados), las organizaciones no gubernamentales de tipo altruista en las que predomina gente que no son profesionales de la salud (como las asociaciones y ligas contra el cáncer), las organizaciones formadas por ciudadanos sin vínculos con las profesiones sanitarias (como nofumadores.org), y otras con fines diversos pero que por su naturaleza o principios pueden mantener posiciones favorables a la prevención, como las de consumidores.

La difusión pública del descubrimiento científico del daño que causaba el tabaco a la salud no desencadenó en España, como lo hizo en otros países, ${ }^{22}$ respuestas 
organizadas proprevención en los años sesenta. Las razones principales para que sucediera de esta forma son el contexto político y social de la dictadura, así como la propiedad estatal de lo que entonces era la empresa monopólica de tabaco (tabacalera). ${ }^{23}$ Con timidez se difunde en los ambientes médicos, en los que entonces el consumo de tabaco es, por cierto, muy elevado. Tras el cambio democrático, desde mediados de los años ochenta van emergiendo personalidades vinculadas con las profesiones sanitarias y las administraciones públicas o con la red asistencial de financiación pública.

Aún resulta impropio hablar de un movimiento de prevención, y la mayoría de las personalidades que expresan de forma visible posiciones respecto al tabaco es experta en un tema $u$ otro, pero sin una visión global. La conciencia de la necesidad de coordinación independiente de la administración se va consolidando a principios de los años noventa, a medida que se amplían las iniciativas de prevención, todo lo cual genera las condiciones para articular una organización global que será el Comité Nacional para la Prevención del Tabaquismo (CNPT). ${ }^{24}$

Las bases para la creación del CNPT se sentaron en una reunión informal de los participantes españoles en la $9^{\text {a }}$ Conferencia Internacional sobre el Tabaco y la Salud de París, en octubre de 1994. En su transcurso se constata la débil presencia española y la frustración por los escasos progresos conseguidos desde la Conferencia anterior, de 1992, en Buenos Aires; frente a ello se propone crear una plataforma de coordinación. Para concluir, se establecen las bases para fundar y constituir de manera formal el CNPT, en 1996. Así, la nueva organización se conformó básicamente como una alianza o coalición de organizaciones ya existentes, que mantenían su actividad de prevención del tabaquismo, que al principio se circunscribía a sociedades científicas o de profesionales sanitarios. Tras un inicio dubitativo y con cierta dispersión, el CNPT comenzó un proceso a largo plazo orientado a influir en las políticas públicas relacionadas con el tabaco. Para ello, se concentró en introducir el tabaco en la agenda política y en definir de forma integral las acciones de prevención prioritarias en España. Como medida inicial, este esfuerzo se concretó en la elaboración del Libro Blanco sobre el tabaquismo en España. ${ }^{25}$ El Libro Blanco marca un hito: movilizó los esfuerzos de muchas personas clave, sintetizó la información, definió prioridades y circuló con amplitud entre el público y la prensa (gracias a su disponibilidad en internet). A continuación, el CNPT contribuyó a generar documentos estratégicos sobre otros aspectos clave, como la publicidad o lo fiscal, y a difundir la necesidad de ejercer influencia en las políticas públicas. ${ }^{26}$ Mediante seminarios y expertos, el CNPT desarrolló documentos de posición sobre políticas clave: espacios sin humo, publicidad, fiscalidad, tratamiento y las presuntas campañas "de prevención" para jóvenes generadas por la propia industria tabaquera (material disponible en www.cnpt.es).

En su interacción con el Ministerio, el CNPT ha influido en la adopción de normas en España, contribuyendo a hacerlas más preventivas tanto para mejorar la normativa sobre el tabaco en los medios de transporte en 1999, como en la transposición de la Directiva Europea de Productos en 2002, y en especial, en la aprobación del Plan Nacional de Prevención del Tabaquismo de 2003 y la ley de medidas sanitarias frente al tabaquismo de 2005, que concreta aspectos regulatorios previstos en el Plan. ${ }^{27}$ Asimismo, diversas Administraciones regionales (autonómicas) consultaron al CNPT o sus miembros para la elaboración de planes locales sobre el tabaco. Desde el CNPT se produjeron acciones de lobbying (cabildeo) para el control del tabaquismo ante los miembros españoles del Parlamento Europeo con el objeto de influir en las Directivas de Publicidad en 1998 y 2002, así como en las Directivas de Productos y de Fiscalidad discutidas en 2001-02, y ante diputados y senadores (como, por ejemplo, con el envío de 15000 tarjetas postales con información sobre los efectos del tabaco, figura 2) para favorecer la aprobación de la ley de 2005, que se comentan en detalle a continuación.

\section{Un momento crucial: la nueva ley de medidas sanitarias frente al tabaquismo}

A finales de 2004 tiene lugar una intensa actuación encaminada a concretar las acciones reguladoras del incipiente Plan Nacional para la Prevención del Tabaquismo en una ley que permitiera un salto cualitativo, aprovechando la transposición legal de la directiva europea de publicidad (cuadro II). Esto se consigue con

\section{Cuadro I \\ Prioridades definidas por el Comité Nacional de Prevención del Tabaquismo, año 2000}

Política fiscal que lleve a precios más disuasorios Regulación rigurosa de la promoción y la publicidad Ampliación efectiva de los espacios sin humo Expansión de las iniciativas comunitarias de prevención Ayuda para los fumadores que quieren dejar de fumar Desarrollo de las acciones de información al público Consolidación de una red para la prevención

Fuente: Referencia 26 
la Ley 28/2005 de medidas sanitarias frente al tabaquismo y reguladora de la venta, el suministro, el consumo y la publicidad de los productos del tabaco, 28,29 en un clima de amplio consenso político y mediático. El objetivo declarado por esta ley es evitar el inicio del consumo de tabaco, en especial entre los jóvenes, garantizar el derecho de los no fumadores a respirar aire sin humo del tabaco y hacer más fácil el abandono de este hábito a las personas que lo deseen. Los temas principales que incluye son la prohibición de la publicidad del tabaco y la limitación de su consumo en centros de trabajo y espacios públicos cerrados.

La nueva ley sustituyó a la normativa previa en España, que era una de las más permisivas de la Unión Europea en temas como la venta de tabaco, limitación de la publicidad y restricciones de lugares de consumo. De inicio, la ley se planteó para establecer la
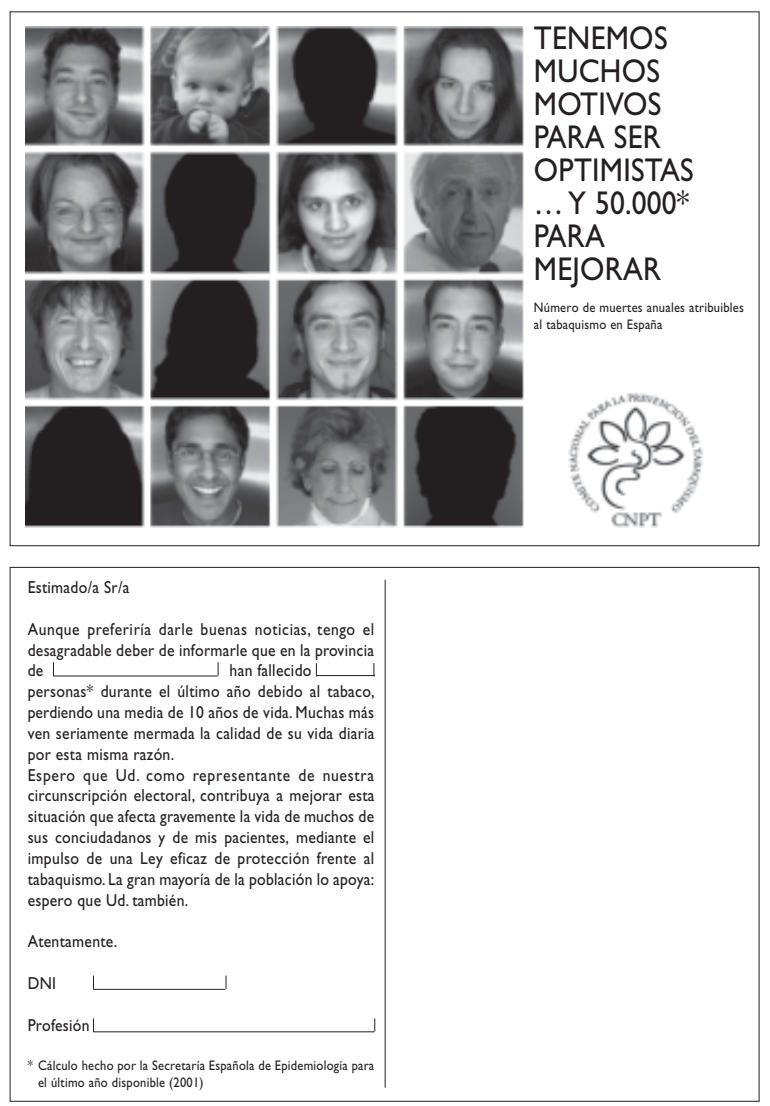

Fuente: Comité Nacional para la Prevención del Tabaquismo

Figura 2. TARJetas (ANVERSO Y REVERSO) ENVIADAS A Los DIPUTADOS Y SENADORES ESPAÑOLES A FAVOR DEL PROYECTO DE LEY DE PREVENCIÓN Y CONTROL DEL TABAQUISMO directiva europea de publicidad que la limitaba en medios transfronterizos (como radio o prensa), pero se amplió a otros temas previstos en el Plan Nacional de 2003 y se presentó tras la ratificación por parte del gobierno español, en diciembre de 2004, del Convenio Marco de la OMS para el Control del Tabaco. ${ }^{30}$ La ley fija limitaciones a la venta y suministro de los productos derivados del tabaco, a su consumo y a la publicidad, promoción y patrocinio. Esta ley comporta la implantación de la prohibición general de fumar en los lugares cerrados de trabajo, con algunas excepciones en el sector de hostelería, restauración y ocio, todo lo cual implica un gran salto adelante.

\section{Prioridades del movimiento de prevención del tabaquismo para el futuro}

Sin duda alguna, la Ley 28/2005 representa el punto culminante de un largo camino para el movimiento de prevención en España; ${ }^{31}$ sin embargo, no es sino un hito más en ese recorrido, pues todavía restan numerosas acciones por emprender. Es asunto reconocido que en la etapa actual corresponde efectuar una evaluación efectiva del impacto de la ley en el consumo de tabaco, en especial en jóvenes y mujeres, en términos tanto de abandono como de retraso o prevención del consumo en estos colectivos, como de protección ante el humo ambiental del tabaco de la población no fumadora -cerca de 70\% en España, en la actualidad. Además, hay que contrarrestar a la industria tabaquera que está impulsando acciones contrarias a la ley, en particular en el sector del ocio, para lo que cuenta con la entusiasta aprobación de las patronales de la restauración y hostelería. Para concluir, se requiere neutralizar una nueva amenaza: parece que la lógica dialéctica gobierno-oposición está llevando a algunos gobiernos regionales detentados por el principal partido de oposición al gobierno central a emprender acciones que rompen el consenso político conseguido al legislar en las Cortes.

Está claro pues que falta mucho por hacer. Hoy en día, es necesario generar apoyo social a la nueva regulación, y redoblar esfuerzos de sensibilización y educación del público. En primer lugar, se requieren acciones en un ámbito general, con actuaciones desde las estructuras de la propia Administración, como por ejemplo, se hizo (con éxito) en Irlanda con la creación de la Oficina para el Control del Tabaco que aportó y preparó el camino años antes de la entrada en vigor de la legislación en aquel país, ${ }^{32}$ y con acciones de organizaciones no gubernamentales y sindicatos que promuevan la concienciación social de determinados colectivos en apoyo genérico y por un efectivo cum- 


\section{Cuadro II}

\section{Argumentos a favor y en contra de la Ley esgrimidos a lo largo del PRoceso español}

\begin{abstract}
A favor
El humo ambiental de tabaco es un cancerígeno para el cual no hay un umbral seguro de exposición

La publicidad es un factor de inducción del consumo en los menores
\end{abstract}

Sólo las separaciones físicas, completamente compartimentadas y con ventilación independiente, aseguran la protección de los no fumadores

Otros países (Irlanda, Noruega, Italia) han aprobado leyes similares o más estrictas sin perjuicios para la economía

No hay evidencia de que el sector de la hosteleria sufra pérdidas al aplicar las regulaciones

No va a haber una disminución de productividad. Los fumadores adaptarán su patrón de consumo a la legislación

La mayoría de la población (más de 70\% según encuestas del Gobierno) apoya la regulaciones

La aplicación del la Ley no va a provocar conflictos ni enfrentamientos entre la población, como lo demuestra la experiencia de otros países

El aumento de los impuestos al tabaco disminuye el consumo
En contra

Los "muros" van a recluir a los fumadores en "ghettos"

En un mercado maduro la publicidad sólo es un factor de competencia entre marcas para el consumo de adultos

Los sistemas de ventilación son capaces de limpiar el humo de tabaco. Hay "papeleras de humo" capaces de depurar las partículas del tabaco

La mayoría de países permite salas para fumar en las empresas. La negociación entre empresarios y sindicatos es una solución efectiva

La hostelería va a enfrentar grandes pérdidas ( 600 millones de euros y 20000 puestos de trabajo)

Va a disminuir la productividad de las empresas al tener que salir, los fumadores, al exterior

La mayoría de los ciudadanos no está de acuerdo con leyes tan estrictas y prohibicionistas que atentan contra la libertad

Se va a dividir a los ciudadanos porque la sociedad no reclama espacios sin humo

El aumento de la fiscalidad del tabaco aumenta el contrabando y la venta de productos de baja calidad plimiento de la nueva Ley. Se necesitan, además, numerosas y diversas expresiones en un ámbito más concreto: todos los profesionales sanitarios tienen oportunidades de acción en los ámbitos local y sectorial en este terreno.

Por último, se debe tener en cuenta que este es un momento inmejorable para emprender proyectos de investigación evaluativa sobre el impacto de estas medidas en el tabaquismo y en la salud, en la línea de los generados por otras experiencias recientes. ${ }^{33,34}$ Dado que la situación de la epidemia tabáquica en España es peculiar, como se describe más arriba, la evaluación de la utilidad de las diversas medidas en este momento puede ser valiosa para orientar las acciones en contextos que se encuentran en situaciones comparables a la española hasta hace pocos años, como la que impera en los países de Latinoamérica.

\section{Agradecimientos}

Los autores agradecen la financiación del Instituto de Salud Carlos III a través de las Redes Cooperativas de Investigación en Salud Pública (RCESP C03/09) y en Cáncer (RTICCC C03/10) y del Fondo de Investigación Sanitaria (proyectos PI 020981 y PI052072) del Ministerio de Sanidad y Consumo del Gobierno de
España, así como del Departament d'Universitats, Recerca i Societat de la Informació de la Generalitat de Catalunya a través de las ayudas para la Red Temática sobre Prevención del Tabaquismo (2003XT/00090) y el Grupo de Investigación en Epidemiología, Prevención y Control del Tabaquismo (2005SGR00646).

\section{Referencias}

I. Fernandez E, Gonzalez JR, Borras JM, Sanchez V, Moreno V, Peris M. Recent decline in cancer mortality in Catalonia (Spain). A joinpoint regression analysis. Eur J Cancer 200 I;37(I7):2222-2228.

2. Villalbí JR, Pasarín MI, Nebot M, Borrell C. Mortalidad por cáncer de pulmón: inicio del declive en los varones de Barcelona. Med Clin (Barc.) 200I; I I7(19):727-73I.

3. Regidor E, Gutiérrez-Fisac JL, Calle ME, Otero A. Patrón de mortalidad en España, 1998. Med Clin (Barc.) 2002;1 18:13-15. 4. González Enríquez J, Banegas Banegas JR, Rodríguez Artalejo F, Díez Gañán L,Villar Álvarez F. La mortalidad atribuible al tabaquismo comienza a descender en España. Med Clin (Barc.) 2005;124(20):769-77I.

5. López MJ, Pérez M, Schiaffino A. Mortalidad atribuible al tabaquismo pasivo en España (2002). Gac Sanit 2005; I9(Supl I):3I 26.

6. Fernández E, Schiaffino A, García M, Saltó E,Villalbí JR, Borrás JM. Prevalencia del consumo de tabaco en España entre 1945 y 1995. Reconstrucción a partir de las Encuestas Nacionales de Salud. Med Clin (Barc.) 2003;120(I):14-16. 
7. Fernandez E, Schiaffino A, Borras JM, Shafey O,Villalbi JR, La Vecchia C Prevalence of cigarette smoking by birth cohort among males and females in Spain, 1910-1990. Eur J Cancer Prev 2003;12: 57-62. 8. Shafey O, Fernandez E, Thun M, Schiaffino A, Dolwick S, Cokkinides V. Case studies in international tobacco surveillance: cigarette advertising and female smoking prevalence in Spain, 1982-1997. Cancer 2004;100:1744-1749.

9. Schiaffino A, Fernandez E, Borrell C, García M, Borras JM. Gender and educational differences in smoking initiation rates in Spain from 1948 to 1992. Eur J Public Health 2003; 13:56-60.

10. Fernandez E, Schiaffino A, García M, Borras JM. Widening social inequalities in smoking cessation in Spain, 1987-1997. J Epidemiol Community Health 200 I;55:729-730.

II. Fernández E, Schiaffino A, Peris M. Tabaquismo en mujeres: un problema de salud emergente. Enfermedades Emergentes 2001;3:184-190.

12. Fernandez E, Schiaffino A, Borrell C, Benach J,Ariza C, Ramon JM et al. Social class, education and smoking cessation: long term follow-up of patients treated at a smoking cessation unit. Nicotine Tob Res 2005;8:29-36.

13. Lopez AD, Collishaw NE, Piha T.A descriptive model of the cigarette epidemic in developed countries. Tob Control 1994;3:242-247.

14. Rogers EM. Diffusion of innovations. New York: The free Press, 1983. 15. Villalbí JR, López V. La prevención del tabaquismo como problema político. Gac Sanit 200I;15: 265-272.

16. Granero L,Villalbi JR, Gallego R. ¿Quién se opone a la prevención? Un mapa de los actores pro-tabaco en España. Gac Sanit 2004;18:374-379.

17. Joosens L. Estrategias de presión para el avance de políticas de prevención del tabaquismo. En:Villalbi JR, Ariza C (coords.). El tabaquismo en España: situación actual y perspectivas para el movimiento de prevención. Barcelona: Comité Nacional de Prevención del Tabaquismo y Sociedad Española de Salud Pública y Administración Sanitaria, 2000; 23-28. Disponible en: http://www.cnpt.es/fr_info.html. I8.Villalbi JR, Córdoba R. El control del tabaquismo y el movimiento de prevención. Adicciones 2005; 17:297-300.

19. Burson-Marsteller. Social acceptability program. Initial recommendations for Spain. Madrid, January 1992. Disponible en: 2500 I20653/0669. URL: http://www.pmdocs.com/PDF/ 2500I20653_0669_0.PDF

20. Soto-Mas F,Villalbí JR, Granero L, Jacobson H, Balcázar H. Los documentos internos de la industria tabaquera y la prevención del tabaquismo en España. Gac Sanit 2003;17(Supl 3):9-14.

2I.Villalbí JR, Salvador T, Granero L. El movimiento de prevención y su organización: papel del Comité Nacional para la Prevención del
Tabaquismo. En: Jiménez-Ruiz CA, Fagerstrom KO eds. Tratado de tabaquismo. Madrid: Grupo Aula Médica 2004:57I-580.

22. Department of Health and Human Services, Office on Smoking and Health. Reducing the health consequences of smoking: 25 years of progress. A report of the Surgeon General. Rockville, MD; 1989. 23. Villalbí JR. El tabaco como problema de salud pública. En: Cabasés JM, Villalbí JR,Aibar C. Invertir para la salud: prioridades en salud pública. Informe SESPAS 2002.Valencia: Generalitat Valenciana y Sociedad Española de Salud Pública y Administración Sanitaria; 2002:I I3-29. Disponible en: http://www.sespas.es/informe2002/cap07.pdf. 24.Villalbí JR. Trabajando para el control del tabaco desde el Comité Nacional de Prevención del Tabaquismo. Prevención del Tabaquismo 200I; 3(Supl I): SI6-17.

25. Becoña $E$ (coord.). Libro blanco sobre el tabaquismo en España. Barcelona: Glosa, 1999. Dispponible en : http://www.cnpt.es/fr_info.html. 26.Villalbí JR, Ariza C (coords.). El tabaquismo en España: situación actual y perspectivas para el movimiento de prevención. Barcelona: Comité Nacional de Prevención del Tabaquismo y Sociedad Española de Salud Pública y Administración Sanitaria; 2000. Disponible en: http:// www.cnpt.es/fr_info.html.

27. Saiz I, Rubio J, Espiga I, Alonso B, Blanco J, Cortés M, et al. Plan Nacional de Prevención y Control del Tabaquismo. Rev Esp Salud Pública 2003;77:441-473.

28. Ley $28 / 2005$ de 26 de diciembre, de medidas sanitarias frente al tabaquismo y reguladora de la venta, el suministro, el consumo y la publicidad de los productos del tabaco. Boletín Oficial del Estado 309 de 27 de diciembre de 2005; 4224I-50. Disponible en http:// www.cnpt.es/Ley\%2028-2005.pdf

29. Fernandez E. Spain: going smoke free. Tob Control 2006;15(2):79-80. 30. Peruga A. El Convenio Marco para el Control del Tabaco: respuesta a la globalización de una epidemia comunicada. Gac Sanit 2004;18:343-345. 31.Villalbí JR. De las propuestas del movimiento de prevención al consenso político: la ley de medidas sanitarias contra el tabaquismo. Gac Sanit 2006;20: I-3.

32. Allwright S, McLaughlin JP, Murphy D et al. Report on the health effects of environmental tobacco smoke (ETS) in the workplace. Dublin: Health and Safety Authority/Office of Tobacco Control; 2003.

33. Sargent RP, Shepard RM, Glantz SA. Reduced incidence of admissions for myocardial infarction associated with public smoking ban: before and after study. BMJ 2004;328: 977-983.

34. Allwright S, Paul G, Greiner B, Mullally BJ, Pursell L, Kelly A et al. Legislation for smoke-free workplaces and health of bar workers in Ireland: before and after study. BMJ 2005;33 I (7525): I I I - I I 23. 